\title{
Probing Electric Field in an Enclosed Field Mapper for Characterizing Metamaterials
}

\author{
Sucheng Li, Chendong Gu, Yadong Xu, Shahzad Anwar, Weixin Lu, \\ Zhi Hong Hang, Bo Hou, and Huanyang Chen \\ College of Physics, Optoelectronics and Energy, Soochow University, 1 Shizi Street, Suzhou 215006, China \\ Correspondence should be addressed to Bo Hou; houbo@suda.edu.cn
}

Received 12 June 2014; Accepted 15 August 2014; Published 27 August 2014

Academic Editor: Atsushi Mase

Copyright (c) 2014 Sucheng Li et al. This is an open access article distributed under the Creative Commons Attribution License, which permits unrestricted use, distribution, and reproduction in any medium, provided the original work is properly cited.

\begin{abstract}
Spatially mapping electromagnetic fields in the quasi-two-dimensional field mapper (a parallel plate waveguiding system; Justice et al. (2006)) for characterizing metamaterial devices, especially those integrating the metal boundary, may encounter troubles including electromagnetic leakage caused by the air gap and energy guiding along finitely high metal walls. To eradicate them, a moving contact approach is proposed. The physical air gap between the mobile metal walls and the stationary upper plate of the mapper is closed, while their relative movement is still allowed during the field mapping. We demonstrate the method of closing the gap by mapping the E-field distribution in a rectangular waveguide.
\end{abstract}

\section{Introduction}

Over a decade ago, the investigation of metamaterials was initiated by the first realization of negative refraction index in microwave frequencies that emerges from simultaneously negative permeability and permittivity and the concept of perfect lens that utilizes negative refraction to obtain subdiffraction-limit resolution [1-6]. Recently, the introduction of transformation optics [7-9] has intensely boosted the investigation on various complex electromagnetic (EM) materials expanding from negative index metamaterials to zero-index metamaterials [10-12] and to extremely anisotropic metamaterials [13-17], because the full control of whole constitutive tensors of metamaterials will bring about various exotic EM phenomena, including artificial magnetism [18, 19], cloaking [20], and illusion [21-24].

The experimental verifications of these exotic wave phenomena frequently need spatial mapping of EM fields inside metamaterials or functional devices. This task is conveniently done with a quasi-two-dimensional (quasi-2D) field mapper in microwave frequencies which is firstly developed by Justice et al. to characterize negatively refracted fields [25]. Other fascinating wave propagations, such as cloaking [20], perfect imaging [26], and topologically EM state [27], and various transformation devices [28-31] have been visualized sequentially in this setup, and the mapped fields have been seen to agree well with the numerical simulations which are typically the simplified $2 \mathrm{D}$ theoretical models reduced from the original three-dimensional problems. The field mapping apparatus consists of a parallel plate waveguiding chamber, where the lower conducting plate (aluminum) rests on the translational stage and carries the metamaterials to move along two orthogonal directions (e.g., the $x-y$ plane) while the upper conducting plate (aluminum) is stationary and has a tiny hole to allow the insertion of the probing antenna and the detection of the electric field (E-field), as schematically drawn in Figure 1(a). However, during the spatial mapping, a $\sim 1 \mathrm{~mm}$ gap between the top surface of the metamaterials and the upper plate has to be maintained in order to facilitate the relative movement between them. So, the metamaterial devices as well as the auxiliary boundary materials under test must be finitely high, for example, $10 \mathrm{~mm}$ at X-band frequencies $(8-12 \mathrm{GHz})[25,32,33]$. Consequently, the electric wall or perfect electric conductor (PEC) boundary in the 2D simulations cannot be mimicked, in exact sense, by erecting a finitely high metal wall inside the quasi-2D mapper. 


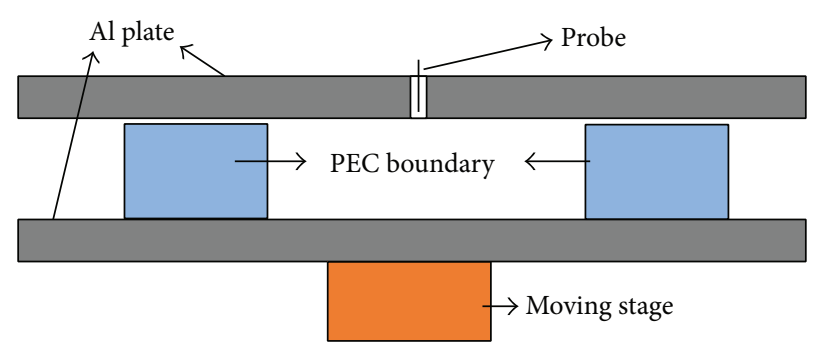

(a)

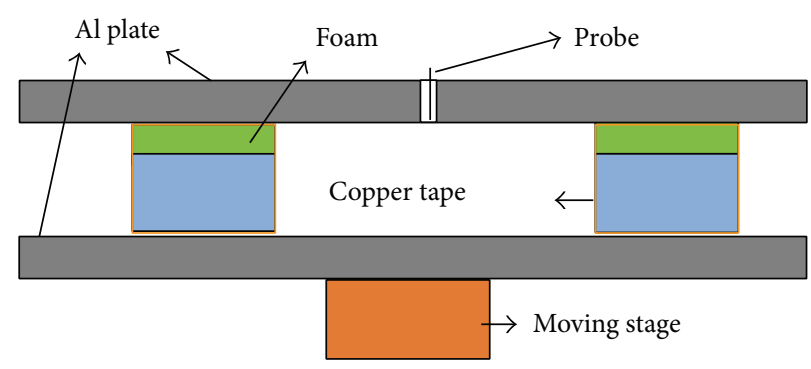

(b)

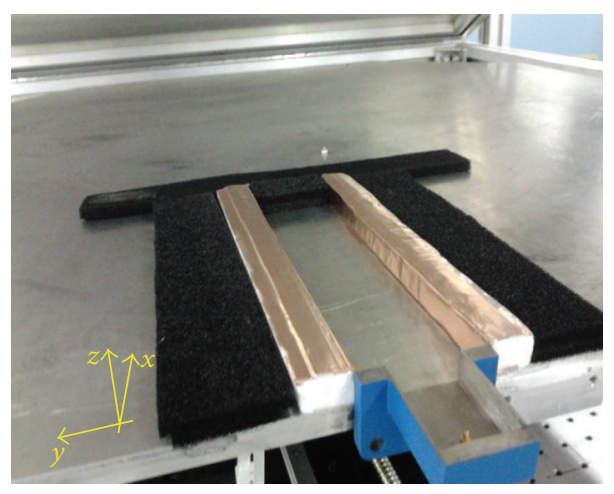

(c)

FIGURE 1: (a) Schematic picture of the quasi-2D electric field mapper with air gap between the metal wall and the upper aluminum plate. (b) Schematic picture of the quasi-2D electric field mapper with the soft contact between the metal wall and the upper aluminum plate. (c) Photo of a section of straight waveguide built with the electrically enclosed metal wall. After the upper plate is in its working position, the waveguide has a rectangular cross section. The lower plate is parallel to the $x-y$ plane.

In this paper, we investigate that the gap can cause inevitable side effects such as EM energy leakage and new energy guiding route along finitely high metal walls, due to which these side effects are undesired for characterizing the metamaterial devices, particularly whose functionality intensely involves or even depends on the metallic boundary. For the first time, we show the spatial mapping of E-fields in an electromagnetically enclosed mapper where the metal wall is contacted physically with the upper plate and at the same time is movable with respect to the upper plate. The moving contact approach has the gap closed and produces the accurate experimental equivalence to the PEC boundary in $2 \mathrm{D}$ numerical models.

\section{Experimental Procedure}

As mentioned above, if the finitely high metal wall acts as the in-plane PEC boundary inside the field mapper, the air gap can cause a leakage of EM energy to unbound exterior regions which are untargeted. Furthermore, it will also make the gap region between the upper plate and the top of metal walls behave as a new waveguiding route, as Efield will be majorly located into the narrow gap, and thus the field characterization of metamaterials under test can be deteriorated. These two problems can be eliminated by closing the gap between the metal wall and the upper plate. In experiments, we cover the top of the metal wall with a soft foam layer and then wrap the foam as well as the metal wall with smooth copper conductive tapes; see Figure 1(b).

Via gently adjusting the height of the lower plate to the position where the metal wall is in physical contact with the upper plate, the air gap is electromagnetically shortened. At the same time, the relative movement between the upper plate and the metal wall is preserved through the foam-capped soft contact between two smooth metallic surfaces (the copper tape and the aluminum plate). A straight waveguide is built in this way, one end excited by a coaxial-to-waveguide adapter that is fixed to the edge of the lower plate and the other end filled in absorbers, shown in Figure 1(c).

\section{Results and Discussions}

In our experimental configuration, a coaxial is inserted into the hole of the upper plate without the tip extruding into the waveguide cavity, which is important to avoid any scratching on the smooth copper tape, and can sense the $E_{\mathrm{z}}$ component inside the chamber. Both the adapter and the coaxial probe are connected with a microwave network analyzer (Agilent $\mathrm{N} 5230 \mathrm{C}$ ) to record the S21 parameter including magnitude and phase. We scanned the straight waveguide region including the metal walls at the spatial resolution $2 \mathrm{~mm} \times 1 \mathrm{~mm}$. To make the comparison, we first did the field mapping in the case of air gap and then scanned the same region after we closed the gap and plotted two cases of results in Figure 2. 


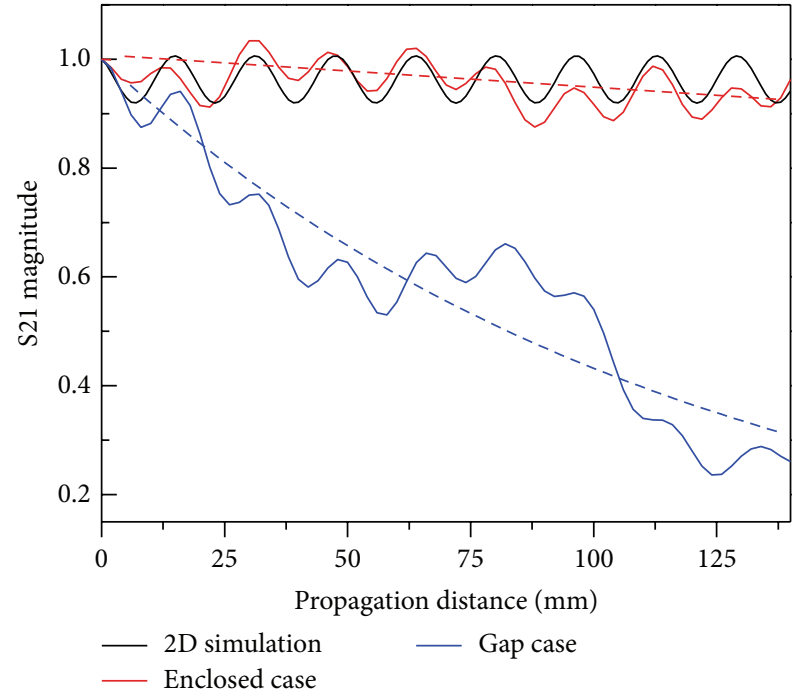

(a)

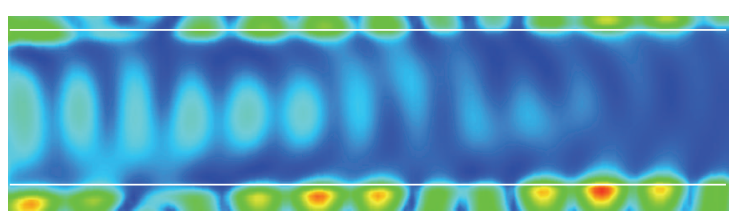

(b)

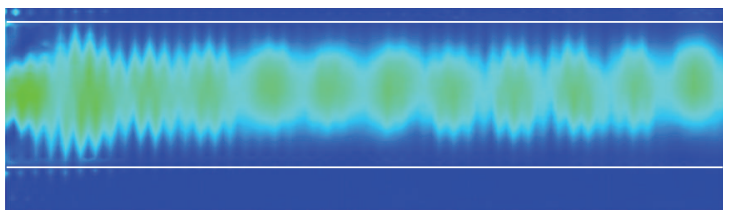

(c)

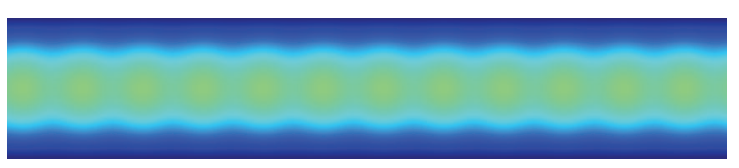

(d)

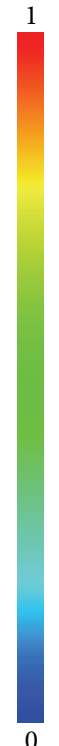

FIgURE 2: (a) The normalized S21 magnitude along the propagation direction. The solid red and blue lines are the measurement for the enclosed and the gap case, respectively, and the black line denotes the 2D simulation. The dash lines represent the exponential fitting of the magnitude decay with a decay length $1613 \mathrm{~mm}$ (red dash line) and $119 \mathrm{~mm}$ (blue dash line). (b) and (c) The measured maps of $E_{z}$ magnitude in the gapped and enclosed case, respectively, where the region sandwiched by the white lines denotes the waveguide cavity. (d) The simulated $E_{z}$ magnitude map.

Figure 2(a) shows the variation of S21 magnitude at $10 \mathrm{GHz}$ versus the propagation distance where the magnitude has been normalized. It is seen that the severe energy leakage takes place along the propagation direction for the gap case (blue lines) with a decay length $119 \mathrm{~mm}$, and the enclosed case (red lines) has negligible propagation loss with significantly larger decay length $1613 \mathrm{~mm}$. In addition, we performed the 2D numerical simulation (COMSOL Multiphysics) for this waveguiding problem where a pair of PEC boundary defines the waveguide, one end is TE10 excitation, and the other end is the scattering boundary. A good agreement between the enclosed case and the simulation can be seen in Figure 2(a), which indicates the EM leakage problem is corrected by our sealing method. Figures 2(b), 2(c), and 2(d) illustrate the spatial distributions of $E_{z}$ magnitude at $10 \mathrm{GHz}$, respectively, for the gap, enclosed, and simulated cases. The region sandwiched by two white lines is the waveguide cavity, and the region outside the lines corresponds to the metal wall. It is seen that the intense E-field is residing over the metal wall in the gap case, Figure 2(b). This is because the electric energy is dominantly stored inside the dimensionally narrower space which has larger effective capacitance, and the wave is guided more efficiently along the metal wall. In contrast, the enclosed case, Figure 2(c), displays a vanishing magnitude of E-field toward the white lines and has no field pattern beyond the white lines, as expected over the PEC boundary in the 2D simulation, Figure 2(d).

By combining the S21 phase, the distribution of the real part of $E_{z}$ can be found in Figure 3, which reveals the gapassociated problems and shows the good agreement between the enclosed case and the $2 \mathrm{D}$ simulation, again. Therefore

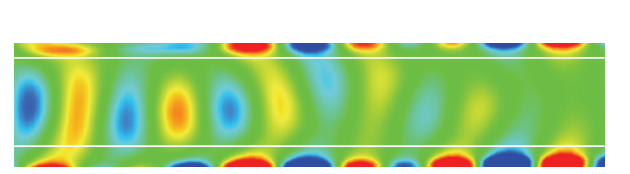

(a)

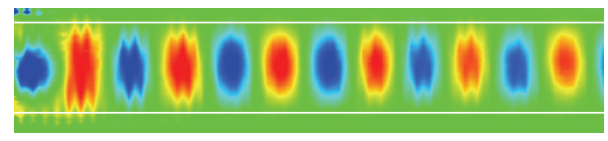

(b)

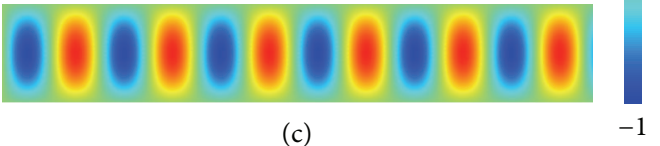

(c)

FIgURE 3: The measured map of $E_{z}$ real part in (a) the gap, (b) the enclosed, and (c) the simulated case, respectively. The region sandwiched by the white lines denotes the waveguide cavity.

the field mapping in the electromagnetically enclosed surrounding gives rise to the experimental results which can be compared directly with the $2 \mathrm{D}$ model simulation.

Although the foam-capped metal wall is gently pressed against the upper plate, the contacting friction due to the relative movement is unavoidable compared to the gapped mapper and thus increases the work loading to the underlying translational motor. The friction might also be responsible for the strip formation in the field map which is noticeable in Figure 2(c). Additionally, as the consequence of the friction, the copper tape needs to be replaced from time to time to maintain the smooth surface. In the future, a soft conducting 
material with more slippery surface will be beneficial much to this technique. For the only purpose of obtaining the field profiles, the gapped mapper may give rise to the reasonably precise results if the gap is controlled to be as small as possible, because the fringe field around the corner of the tiny gap has the negligible influence on the targeted scanning region. However, for characterizing the energy flow in some metamaterial devices, the gap should be closed.

\section{Conclusion}

In conclusion, two problems, energy leakage and new guiding path, have been identified in the quasi-2D field mapper for spatially mapping EM fields of some metamaterial devices, especially those integrated with metal boundaries. They are caused by the air gap facilitating the mapping movement and the resultant finitely high metal walls. We have suggested a moving contact technique that closes the gap between the dynamical metal walls and the stationary upper plate of the mapper without impeding their relative movement. The metal walls under soft contacting enable a direct mimic to the PEC boundary in much simplified 2D numerical models. We demonstrate the technique through mapping the $E_{z}$ field inside a standard waveguide. We believe that our electromagnetically enclosing approach will advance the accurate measurement in characterizing EM fields for metamaterials and transformation media.

\section{Conflict of Interests}

The authors declare that there is no conflict of interests regarding the publication of this paper.

\section{Acknowledgments}

This work was supported by the National Natural Science Foundation of China (Grant nos. 11004147, 11104198, and 11304215), the Natural Science Foundation of Jiangsu Province (Grant nos. BK2010211, BK20130281, and BK20141191), and a Project Funded by the Priority Academic Program Development (PAPD) of Jiangsu Higher Education Institutions.

\section{References}

[1] V. G. Veselago, "The electrodynamics of substances with simultaneously negative values of $\varepsilon$ and $\mu$," Soviet Physics Uspekhi, vol. 10, no. 4, pp. 509-514, 1968.

[2] D. R. Smith, W. J. Padilla, D. C. Vier, S. C. Nemat-Nasser, and S. Schultz, "Composite medium with simultaneously negative permeability and permittivity," Physical Review Letters, vol. 84, no. 18 , pp. $4184-4187,2000$.

[3] J. B. Pendry, "Negative refraction makes a perfect lens," Physical Review Letters, vol. 85, no. 18, pp. 3966-3969, 2000.

[4] N. Fang, H. Lee, C. Sun, and X. Zhang, "Sub-diffraction-limited optical imaging with a silver superlens," Science, vol. 308, no. 5721, pp. 534-537, 2005.
[5] D. R. Smith, J. B. Pendry, and M. C. K. Wiltshire, "Metamaterials and negative refractive index," Science, vol. 305, no. 5685, pp. 788-792, 2004.

[6] Y. Liu and X. Zhang, "Metamaterials: a new frontier of science and technology," Chemical Society Reviews, vol. 40, no. 5, pp. 2494-2507, 2011.

[7] U. Leonhardt, “Optical conformal mapping," Science, vol. 312, no. 5781, pp. 1777-1780, 2006.

[8] J. B. Pendry, D. Schurig, and D. R. Smith, "Controlling electromagnetic fields," Science, vol. 312, no. 5781, pp. 1780-1782, 2006.

[9] H. Chen, C. T. Chan, and P. Sheng, "Transformation optics and metamaterials," Nature Materials, vol. 9, no. 5, pp. 387-396, 2010.

[10] M. Silveirinha and N. Engheta, "Tunneling of electromagnetic energy through subwavelength channels and bends using $\varepsilon$ near-zero materials," Physical Review Letters, vol. 97, no. 15, Article ID 157403, 2006.

[11] R. Liu, Q. Cheng, T. Hand et al., "Experimental demonstration of electromagnetic tunneling through an epsilon-nearzero metamaterial at microwave frequencies," Physical Review Letters, vol. 100, no. 2, Article ID 023903, 2008.

[12] B. Edwards, A. Alù, M. E. Young, M. Silveirinha, and N. Engheta, "Experimental verification of epsilon-near-zero metamaterial coupling and energy squeezing using a microwave waveguide," Physical Review Letters, vol. 100, no. 3, Article ID 033903, 2008.

[13] J. Luo, P. Xu, H. Chen, B. Hou, L. Gao, and Y. Lai, "Realizing almost perfect bending waveguides with anisotropic epsilonnear-zero metamaterials," Applied Physics Letters, vol. 100, no. 22, Article ID 221903, 2012.

[14] P. B. Catrysse and S. Fan, "Routing of deep-subwavelength optical beams and images without reflection and diffraction using infinitely anisotropic metamaterials," Advanced Materials, vol. 25, no. 2, pp. 194-198, 2013.

[15] W. X. Jiang, T. J. Cui, X. M. Yang, H. F. Ma, and Q. Cheng, "Shrinking an arbitrary object as one desires using metamaterials," Applied Physics Letters, vol. 98, no. 20, Article ID 204101, 2011.

[16] W. X. Jiang and T. J. Cui, "Radar illusion via metamaterials," Physical Review E, vol. 83, no. 2, Article ID 026601, 2011.

[17] W. X. Jiang, C. Qiu, T. Han, S. Zhang, and T. J. Cui, "Creation of ghost illusions using wave dynamics in metamaterials," Advanced Functional Materials, vol. 23, no. 32, pp. 4028-4034, 2013.

[18] J. B. Pendry, A. J. Holden, D. J. Robbins, and W. J. Stewart, "Magnetism from conductors and enhanced nonlinear phenomena," IEEE Transactions on Microwave Theory and Techniques, vol. 47, no. 11, pp. 2075-2084, 1999.

[19] T. J. Yen, W. J. Padilla, N. Fang et al., “Terahertz magnetic response from artificial materials," Science, vol. 303, no. 5663, pp. 1494-1496, 2004.

[20] D. Schurig, J. J. Mock, B. J. Justice et al., "Metamaterial electromagnetic cloak at microwave frequencies," Science, vol. 314, no. 5801, pp. 977-980, 2006.

[21] Y. Lai, H. Chen, Z. Zhang, and C. T. Chan, "Complementary media invisibility cloak that cloaks objects at a distance outside the cloaking shell," Physical Review Letters, vol. 102, no. 9, Article ID 093901, 2009.

[22] Y. Lai, J. Ng, H. Chen et al., "Illusion optics: The optical transformation of an object into another object," Physical Review Letters, vol. 102, no. 25, Article ID 253902, 2009. 
[23] H. Chen, B. Hou, S. Chen, X. Ao, W. Wen, and C. T. Chan, "Design and experimental realization of a broadband transformation media field rotator at microwave frequencies," Physical Review Letters, vol. 102, no. 18, Article ID 183903, 2009.

[24] Y. Xu, S. Du, L. Gao, and H. Chen, "Overlapped illusion optics: a perfect lens brings a brighter feature," New Journal of Physics, vol. 13, Article ID 023010, 2011.

[25] B. J. Justice, J. J. Mock, L. Guo, A. Degiron, D. Schurig, and D. R. Smith, "Spatial mapping of the internal and external electromagnetic fields of negative index metamaterials," Optics Express, vol. 14, no. 19, pp. 8694-8705, 2006.

[26] Y. G. Ma, S. Sahebdivan, C. K. Ong, T. Tyc, and U. Leonhardt, "Evidence for subwavelength imaging with positive refraction," New Journal of Physics, vol. 13, Article ID 033016, 2011.

[27] Z. Wang, Y. Chong, J. D. Joannopoulos, and M. Soljačić, "Observation of unidirectional backscattering-immune topological electromagnetic states," Nature, vol. 461, no. 7265, pp. 772-775, 2009.

[28] Y. G. Ma, C. K. Ong, T. Tyc, and U. Leonhardt, "An omnidirectional retroreflector based on the transmutation of dielectric singularities," Nature Materials, vol. 8, no. 8, pp. 639-642, 2009.

[29] Q. Cheng, W. X. Jiang, and T. J. Cui, "Spatial power combination for omnidirectional radiation via anisotropic metamaterials," Physical Review Letters, vol. 108, no. 21, Article ID 213903, 2012.

[30] Q. Wu, X. Feng, R. Chen et al., "An inside-out Eaton lens made of H-fractal metamaterials," Applied Physics Letters, vol. 101, no. 3, Article ID 031903, 2012.

[31] C. Gu, K. Yao, W. Lu et al., "Experimental realization of a broadband conformal mapping lens for directional emission," Applied Physics Letters, vol. 100, no. 26, Article ID 261907, 2012.

[32] Q. Cheng, W.X. Jiang, and T. J. Cui, "Radiation of planar electromagnetic waves by a line source in anisotropic metamaterials," Journal of Physics D: Applied Physics, vol. 43, no. 33, Article ID 335406, 2010.

[33] Q. Cheng, H. F. Ma, and T. J. Cui, "Broadband planar Luneburg lens based on complementary metamaterials," Applied Physics Letters, vol. 95, no. 18, Article ID 181901, 2009. 

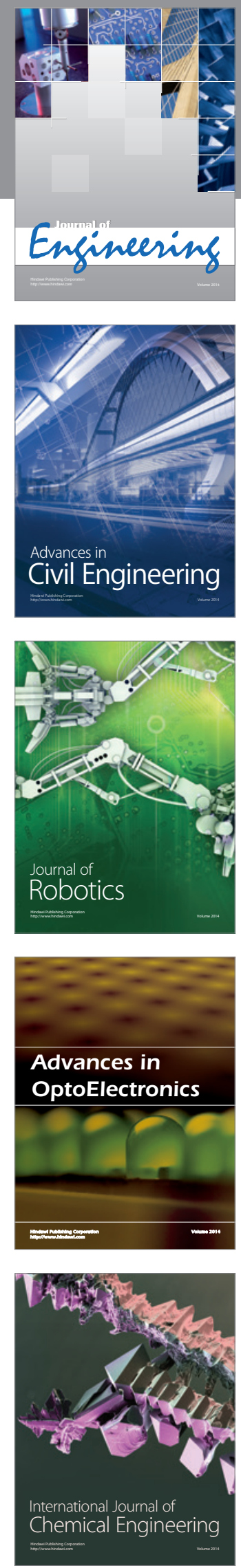

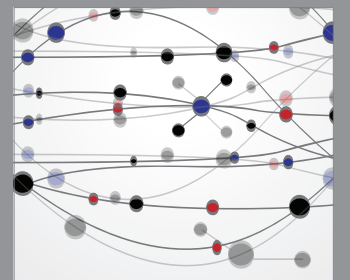

The Scientific World Journal
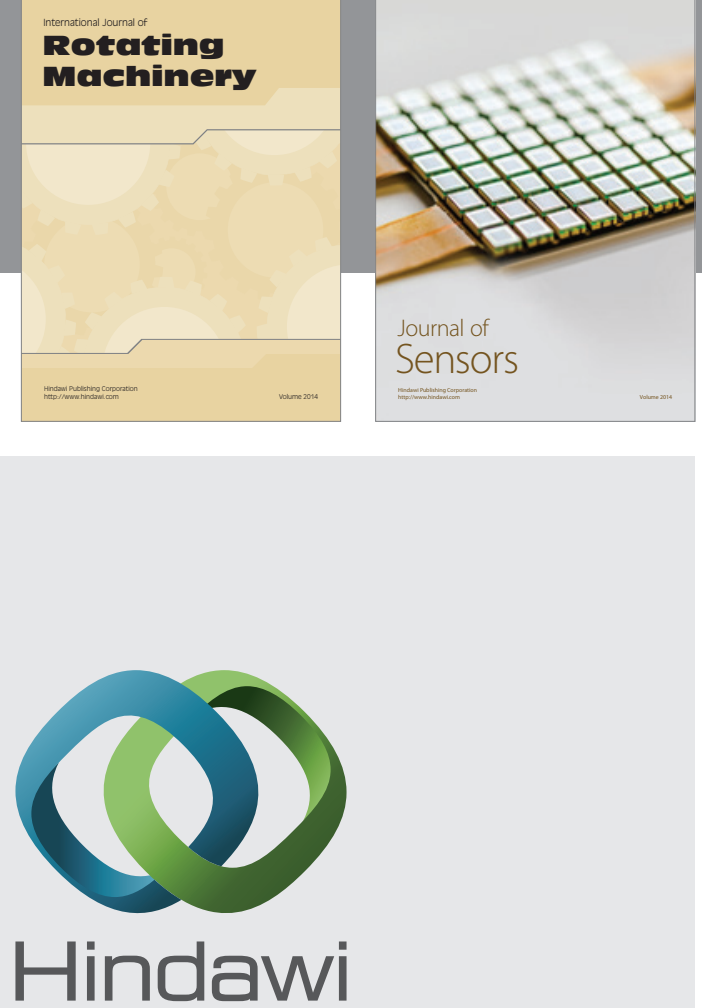

Submit your manuscripts at http://www.hindawi.com
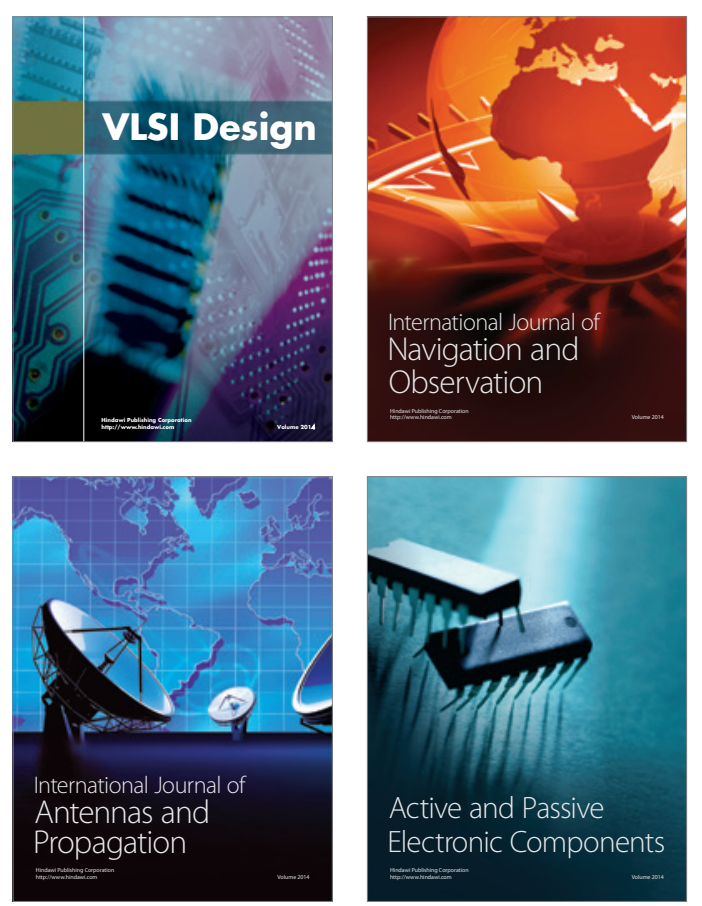
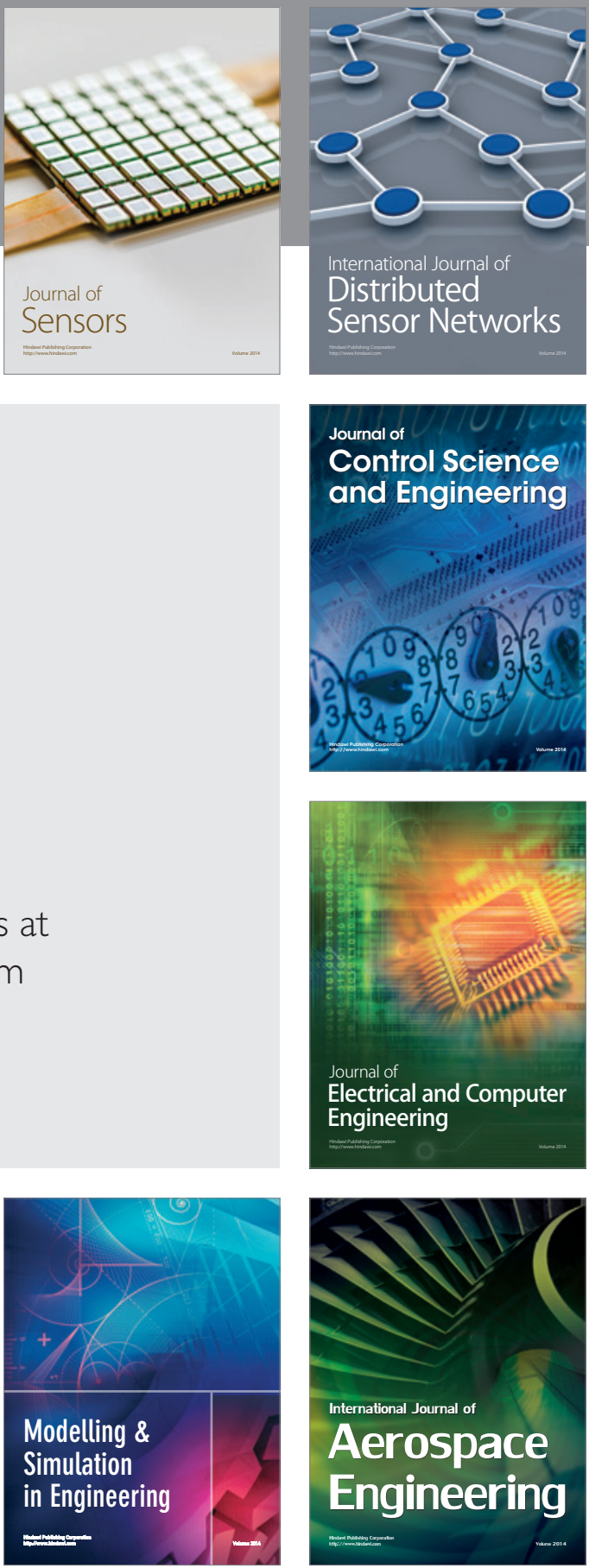

Journal of

Control Science

and Engineering
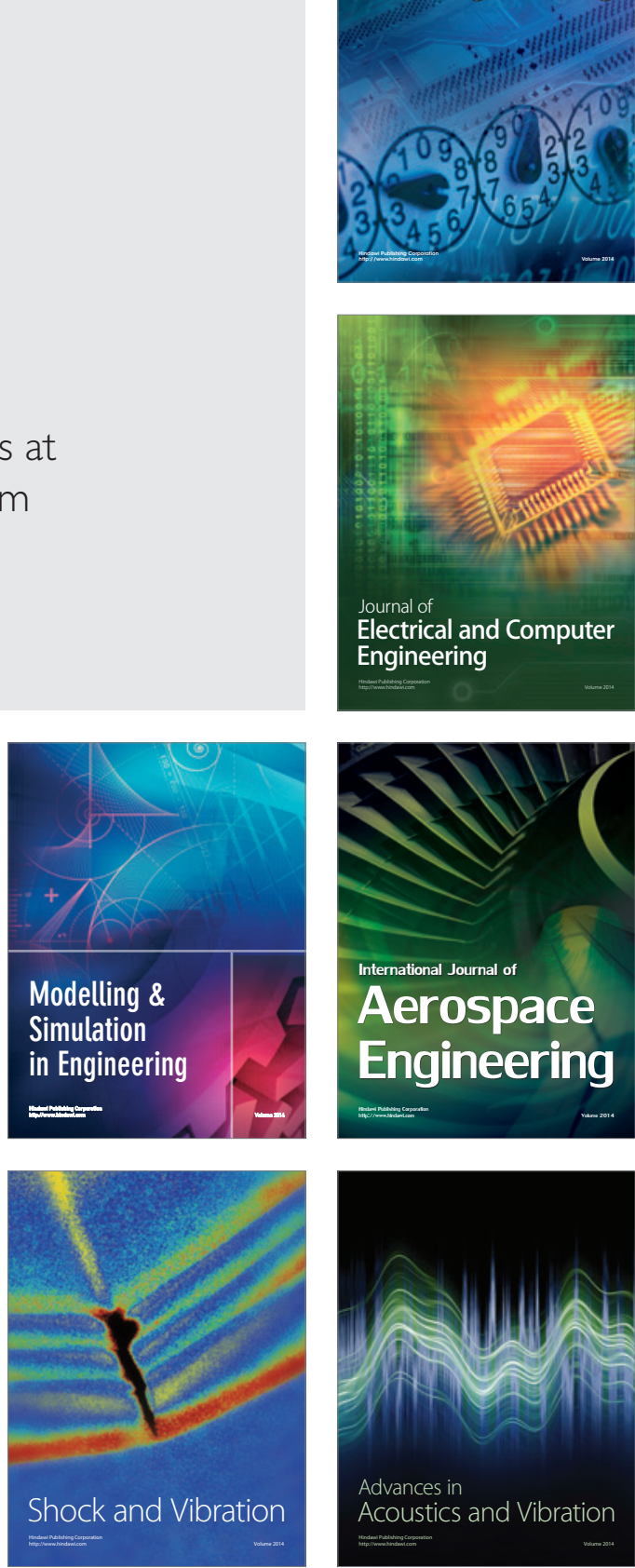